\title{
APUNTES SOBRE EL PROCESO CREATIVO
}

\section{RESUMEN}

En este artículo se aborda el proceso creativo considerando varios de los factores que lo producen, facilitan y empobrecen. Partiendo de la base de que la creatividad es fruto de un proceso de raciocinio arraigada en el esfuerzo por suplir necesidades materiales y espirituales, podemos ver cómo las distintas clases sociales se ven enfrentadas a procesos creativos muy distintos. Además podemos analizar cómo el uso de drogas genera un efecto de frustración creativa y a partir de la diferencia entre el pensamiento convergente y el pensamiento divergente, ver al proceso creativo como un mero acto reiterativo, o como un acto de innovación crítica donde converge la creatividad con la superación de la egolatría.

Palabras Clave: Proceso creativo, pensamiento divergente, funciones del pensamiento, innovación.

\section{NOTES ABOUT THE CREATIVE PROCESS}

\begin{abstract}
This article addresses the creative process considering several factors that produce, facilitate and hinder it. Starting on the principle of creativity being the result of a reasoning process motivated by the effort towards supplying material and spiritual needs, it presents some observations on how different social classes experience contrasting creative processes. Also, the paper offers an analysis on how the use of drugs produces creative frustration effects. Based on the difference between convergent thinking and divergent thinking, the author conceives the creative process as a basic reiterative act or as a critical innovation act where creativity and overcoming of egotism converge.
\end{abstract}

Keywords: creative process, divergent thinking, convergent thinking, thinking functions, innovation

\section{[ FABIO E. MEDELLÍN VARGAS ]}

Diseñador Gráfico (Universidad Nacional de Colombia) y Realizador Audiovisual. Facultad de ciencias de la Comunicación, UNIMINUTO.

fmedellin旬uniminuto.edu.

Recibido: 28 de mayo de 2013

Aceptado: 12 de Julio de 2013

EL PROCESO CREATIVO

\section{Introducción}

Para comenzar es necesario clarificar algunos puntos:

1 Siempre que hablamos de creatividad no estamos - hablando de un producto, eso sería lo creado, es decir hemos creado ese producto; y la creatividad es el proceso por medio del cual se llegó a un producto cualquiera que éste sea.

7 También dejemos claro que algunos tienen más - habilidad en algunas de las fases de ese proceso y otros en otras; es decir sólo algunos pueden llegar a manejar todo el proceso. Hay algunas personas que por muchos motivos, por ejemplo no tuvieron un buen desarrollo de la motricidad fina, de su manualidad, allá en la infancia, son incapaces de hacer una obra manual, pero si los ponen a generar, son capaces de llevar al boceto grandes ideas; hay personas cuya timidez les impide presentarse ante el público, pero son capaces de generar, de idear los poemas, las canciones, los discursos más hermosos del mundo; hay muy pocas personas que son capaces de abordar todo ese proceso que empieza en una idea y termina en un objeto o producto; este proceso es lo que se llama creatividad: tomar una idea evolucionarla, trabajarla, desarrollarla y llevarla a un producto final, casi siempre en un objeto y en algunas ocasiones en un hecho perceptible; de todas maneras siempre llegando a algo que sea captable por los demás.

Si uno escribe poemas, cartas o artículos, pero - no los muestra, no existen como proceso creativo porque no se han completado, ya que el objeto no ha llegado a los demás; es decir todavía no son un producto completo con un espectador; mucho más si lo analizamos desde la comunicación, pues no han cumplido su 
proceso completo mientras no lleguen a un público; y ese público no puedo ser yo mismo, porque no tengo la capacidad de distanciamiento suficiente, para observar ese producto con la crítica objetiva seria; es decir, o bien soy demasiado crítico con lo que hago, no creo en lo mío, o bien soy demasiado laxo con lo que hago.

\section{CREATIVIDAD}

Todos nacemos creativos porque todos nacemos humanos; es decir a nosotros, como seres racionales, nos diferencia del resto de los seres animales, el hecho de poder raciocinar, evaluar, criticar e interpretar las cosas, lo que nos permite solucionar de manera diferente los problemas que se nos presentan; a ese proceso se le llama raciocinar, y esa razón es el fundamento básico de la creatividad.

La creatividad es, por lo tanto, ese proceso por el cual partimos de una idea y construimos otra, otra que se evidencia en algo percibido por los demás, porque les soluciona algún problema o les suple alguna necesidad.

Esta creatividad que se deja ver cuando nos enfrentamos a la solución de problemas o necesidades, y como todos buscamos permanentemente tener un estado inalterado, de tranquilidad absoluta, explica que el niño o la persona de las clases bajas que tiene muchas necesidades físicas concretas: sufrirá de hambre, de frío, de dolor, etc., llegue a productos muy perceptibles, mientras que la persona de la clase alta que tiene otro tipo de necesidades, pues eso del hambre, del frío o del dolor la persona de clase alta lo tiene solucionado, pero a él le falta afecto, cariño, amor, reconocimiento, compañía, etc., es decir tiene necesidades espirituales, llega a productos poco perceptibles. Es decir las necesidades diferentes llevan a procesos creativos diferentes, y aquel que tiene necesidades terriblemente concretas, llegará a productos muy concretos, mientras aquel que tiene necesidades conceptuales, en el nivel de lo espiritual, llegará a productos sutiles.

Muchos creen que la droga estimula la creatividad, porque parten del principio equivocado de que sus efectos agudizan la percepción, cuando lo único que hacen es alterar las percepciones y hacer sentir la realidad deformada; la droga lo que consigue es desviarla, llevarla hacia caminos que no ayudan a solucionar los verdaderos problemas y cuestionamientos que ellos generan. Como cualquier idea no nace sino de un cuestionamiento de la realidad, producto de un problema o una necesidad, es decir al encontrarme con algo que no me satisface o no conozco de la realidad la cuestiono, pregunto sobre ella, y a partir de ello clarifico las necesidades que me obligan a actuar; es decir el ser humano mientras no sienta una necesidad, no se mueve a actuar, si todo lo tengo, si todo está solucionado, difícilmente me muevo para conseguir aquello que me hace falta, la persona que utiliza drogas termina por generar algo que no le pertenece o no es suyo, por lo tanto creará una obra no reconocida por sí mismo como su propia obra, sino que la hizo el otro, el que tenía su percepción alterada, el que estaba percibiendo una irrealidad: la producida por la droga; existirá allá en su interior, así no lo exprese, un sentimiento de que eso lo hace el otro; de ahí que una gran cantidad de aquellos artistas que para ejercer su arte tiene que estimularse, viven niveles de frustración muy grandes. Cuando uno requiere de elementos artificiales para darse a conocer, es decir hacer perceptibles sus ideas, es que hay falta de reconocimiento de sí mismo; llámese a ese elemento alcohol o cualquier otro tipo de droga.

\section{La persona que utiliza drogas termina por generar algo que no le pertenece o no es suyo, por lo tanto creará una obra no reconocida por sí mismo como su propia obra, sino que la hizo el otro, el que tenía su percepción alterada, el que estaba percibiendo una irrealidad: la producida por la droga}

\section{PENSAMIENTO}

El pensamiento es el producto de pensar, y pensar es razonar; es este raciocinio lo que nos diferencia de los animales; no hay absolutamente ningún animal que raciocine, única y exclusivamente lo puede hacer el ser humano, el hombre. Es decir el único ser que es capaz de analizar, interpretar, valorar y a partir de ahí generar.

Uno de los elementos del raciocinio es el proceso creativo, y esa creatividad, como proceso, conlleva una serie de pasos o de fases que comienzan cuando identificamos una necesidad sentida o inventada, porque a veces nosotros nos inventamos necesidades, la sociedad nos hace inventar necesidades, y evita que clarifiquemos nuestras prioridades para solucionarlas; por eso el crear parte de identificar o plantear las necesidades que tenemos; ese es el primer peldaño para empezar a crear.

Como la creatividad no nace sino de la necesidad, al arte habrán de acceder aquellas personas que: o bien tienen solucionadas sus necesidades concretas de hambre, dolor, 
frío, etc., las básicas, o bien han relegado esas necesidades concretas a un segundo plano por las necesidades espirituales. Cuando hablo aquí de espiritualidad no tiene nada que ver con lo católico, ni lo cristiano, ni lo mormón, etc., hablo de otra esencia que es específicamente la sensibilidad; somos o no sensibles; si somos sensibles tendremos esa capacidad de hacer a un lado o relegar nuestras necesidades concretas, para dar a conocer nuestras necesidades espirituales de comunicación, de afecto, de amor, de cariño, etc., por eso no todos podemos acceder al arte.

Todos los hombres deberían tener sus necesidades básicas solucionadas, para que así todos puedan acceder al arte mismo, para que así sus necesidades espirituales se manifiesten y se viva esa experiencia; como no se puede ejercer esa sensibilidad, el hombre que está limitado por sus necesidades concretas, en la búsqueda de solucionar esas necesidades concretas, terminará siendo completamente insensible; sobre todo porque esas necesidades concretas cuando no se identifican claramente, terminarán siendo un largo listado imposible de solucionarse jamás.

\section{La creatividad es pues, un proceso que se fundamenta en tres elementos: la memoria, la inteligencia y la innovación o invención, también llamada creatividad propiamente dicha .}

La sensibilidad, pues, no es sino la expresión de las necesidades espirituales y la propuesta de solución a esas necesidades espirituales.

La recursividad dará los niveles de creatividad para resolver las necesidades materiales, la sensibilidad dará la solución a las necesidades espirituales.

Esa creatividad requiere para ser mucho más elaborada, mucho más válida, mucho más loable de tres elementos: la memoria, la inteligencia y la creatividad propiamente dicha; es decir cuando se tiene buena memoria, excelente inteligencia y hay una creatividad desbordada, se tiende a ser genio o se es genio; sin embargo puede existir el caso de una persona que solamente tiene creatividad, muy poca inteligencia y nada de memoria.

\section{ELEMENTOS DE LA CREATIVIDAD}

La creatividad es pues, un proceso que se fundamenta en tres elementos: la memoria, la inteligencia y la innovación o invención, también llamada creatividad propiamente dicha.
La memoria es la capacidad para retener, para guardar y traer a la conciencia; nacida de otra función del pensamiento que es la capacidad de percibir.

La inteligencia es la capacidad para analizar, relacionar las experiencias y sacar conclusiones, por ende fijar posiciones; lógicamente es entendible el hecho de que si uno tiene memoria, puede ser más inteligente, porque tiene más experiencias que relacionar, aquel que no tiene memoria puede tener inteligencia, pues relaciona las experiencias inmediatas y saca conclusiones, luego se puede ser inteligente sin tener memoria, para que se sea bastante inteligente, es imprescindible, también, tener una buena dosis de memoria, porque ya no sólo se relaciona lo que se está vivenciando, sino lo que se recuerda, se relaciona todo y se saca una conclusión más elaborada y sustentada; cierro esta definición con el hecho de que quien toma posición, lo hace porque, precisamente, cuando relaciona, saca conclusiones, y es inevitable que con las palabras o con los hechos, dé un punto de vista, y un punto de vista es ya una posición con respecto a las cosas. ¿En dónde no encontramos a la persona inteligente? En donde encontramos a un buen memorista, en aquel que no se compromete con nada, aunque maneje toda la información necesaria para hacerlo.

La innovación es la capacidad para generar, incluso, nuevas preguntas sobre las ya solucionadas, no sólo productos; es lógico que si tengo buena memoria y bastante inteligencia, podré crear mejores productos o preguntas.

\section{MODELOS DE PENSAMIENTO}

La creatividad es una de las funciones del pensamiento, pensar es raciocinar, es concluir a partir de comparar, a partir de relacionar, a partir de evaluar, eso es pensar, concluir; esto implica inevitablemente que existan modelos de pensamiento que hacen personas más creativas, y modelos de pensamientos que hacen personas menos creativas; no se trata, cuando hablo de modelos de pensamiento, de qué se piensa sino cómo se hace; es decir, el 90\% de las personas tienen un modelo de pensamiento que los lleva a sacar conclusiones a priori, conclusiones inmediatas a partir de la simple percepción de los hechos; otros modelos de pensamiento obligarán a que la persona reflexione antes de sacar una conclusión.

Estos dos modelos básicos del pensamiento son: el convergente que concluye a partir de la percepción; recibe la información y saca una conclusión; y el modelo divergente que hace un proceso mucho más largo, en cierta medida 
más complicado porque, si bien es cierto él también llega a conclusiones, lo hace después de haber percibido y analizado; probablemente si es más divergente no solamente analiza, sino que antes de concluir interpreta, para, posteriormente, sí dar su crítica, su elemento de valoración.

El que ha concluido a partir de la percepción, en un $90 \%$ de los casos se equivoca en su conclusión; se dice que su conclusión es apresurada.

Como el pensamiento se expresa en nuestros comportamientos, en nuestras conductas, es decir en nuestras acciones, la vida está hecha de acciones; algunas de ellas se llaman actitudes, otras se llamarán conductas, otras se llamarán comportamientos, pero todas son reflejos del pensamiento; es decir de la personalidad.

Las acciones pueden construir situaciones o hechos, y los hechos se pueden convertir en situaciones, es decir una situación es una sucesión de acciones que se repiten: todos los días salgo de mi casa, tomo el transporte, me quedo en la avenida, voy hasta la oficina, etc., esas acciones repetitivas, constantes, son las situaciones, cuando pasa algo distinto se llama hecho, y cuando los hechos cambian la forma de ser de la persona se llaman sucesos.

El convergente tiende a vivir de situaciones, el divergente tiende a vivir de sucesos; el divergente tiene la mayor seguridad del mundo, el convergente no tiene seguridad, porque nunca estará completo, cuando ya tiene los objetos que buscaba, debe ponerle otros objetos al listado; el divergente tiene mayor seguridad, porque su seguridad es el riesgo, abordar el riesgo, abordar el vivir todas las experiencias; ese divergente sabe que su meta nunca va a terminar, el convergente sabe que su meta se termina pronto, porque se coloca metas realmente concretas, visibles, tangibles.

Generalmente somos convergentes en algunas cosas y divergentes en las otras, es decir para ser creativo no se necesita vestirse de creativo, no se tiene porque ser una persona de blue jeans raídos, de botas hasta la rodilla, etc.

El divergente recoge su información no solamente de un modelo, sino que de varios modelos toma y construye el propio, pero tomar no quiere decir copiar; copiar es tratar de ser igual a otro, y aquel que se viste de creativo, probablemente, nunca ha sido divergente, pues se fija de las cosas más externas, pero no se toma ese trabajo con el pensamiento; por eso podemos encontrar al creativo más interesante, el divergente más impresionante, en aquel que conserva las formalidades de una sociedad.

El principio de la convergencia está en la competencia, el ganar, y el principio de la divergencia está en el compartir; nunca terminaremos de compartir, siempre habrá nuevas posibilidades de compartir, mientras el pensamiento convergente obliga a generar más normas, para controlar así a la competencia.

El armar y sostener una pareja es una de las vivencias más intensa, exigente y difícil; placentera en demasiadas ocasiones, dolorosa también en muchas ocasiones, pero si esa pareja se montase sobre la base de una competencia, no podría existir placer en ningún momento, siempre habría angustias; si esa pareja se monta sobre la base de compartir, qué importa quién esté más adelante o más atrás, los dos siempre unidos, así los demás no los reconozcan, seguirán hasta conseguir lo que quieren. El compartir obliga a tomar posición, porque hay muchas posibilidades de decisión, el competir no permite sino una decisión: "ganar”, luego reduce e imposibilita la toma de posición.

\section{BASES DEL PROCESO CREATIVO}

El proceso creativo, como todo proceso, abordará una serie de fases; esas fases están conformadas por acciones, es decir situaciones, hechos o sucesos perceptibles a través del tiempo y el espacio que van construyendo diferentes posibilidades de seguir adelante; de ahí que exista toda una diferenciación muy clara entre fantasía e imaginación; fantasear es crear en la mente, imaginar es crear en lo concreto; fantasear permanentemente es malísimo, imaginar es maravilloso; generalmente fantaseamos, muy pocas veces imaginamos; el niño, por su falta de conocimiento tiende a fantasear; el adolescente por su falta de conocimiento en campos como el amor, tiende a fantasear con él; el joven por su falta de conocimiento en campos como la figuración, tiende a fantasear con ello; el adulto que ya ha acumulado experiencias imagina mucho.

Se dice que los productos de la creatividad más elaborados, más definidos, se dan después de los 50 años, porque ya se ha reunido la experiencia suficiente para que la fantasía no prime sobre la imaginación; no quiero decir que deje de existir la fantasía, tal vez sólo deja de existir la fantasía en aquel anciano, ya muy anciano, que sólo espera la muerte.

La fantasía del niño será crear su propio mundo, porque no conoce el de la realidad, mucho más el niño de la ciudad que está encerrado en un bloque de apartamentos o un inquilinato. El adolescente fantasea con el amor, con el sexo $y$, lógicamente, fantasea tanto con ese tema que termina confundiendo sexo con amor, y en ese equívoco maneja los mismos términos. El joven fantasea con la construcción de una familia y la figuración, el sentido de la posesión, el ser importante económicamente y socialmente, figurar, aparecer y ser reconocido, no solamente, por los hijos. El hombre maduro pinta, escribe, etc., no quiero decir que antes no lo haya hecho, antes ya lo ha hecho, pero con su infinita 
capacidad de ensoñamiento, es decir de engolosinarse con lo que él mismo hace, de creer que nunca está cometiendo un error, de que lo que hace es perfecto; esos son los niveles de maduración, por eso si un adolescente sigue aplicando criterios de fantasía del niño no ha madurado.

El que imagina, según su edad cronológica y mental, ha de ir plasmando su fantasía en objetos o acciones; mientras esos objetos no se den, la creatividad está anulada y se sigue fantaseando.

\section{FASES DEL PROCESO CREATIVO}

Las fases del proceso creativo culminan con la presentación de un producto perceptible, y esas fases son: la fase de incubación, la fase ilustrativa, y la fase de refuerzo. La fase de incubación es aquella suma de acciones que nos dan claridad sobre cuál es el problema. La ilustrativa es aquella en que reunimos información sobre las formas cómo se ha solucionado antes dicho problema, y conformamos nuestra propuesta. Y la de refuerzo es aquella en que evaluamos los resultados y planteamos los nuevos problemas.

Recordemos entonces que para poder crear se necesita, ya que esto es un proceso, de una serie de condiciones en la persona, condiciones que tienen que ver con el comportamiento en la cotidianidad; en esa cotidianidad, en cada una de esas acciones es que se demuestra la capacidad creativa; cómo se piensa y cómo se representa esa manera de pensar. Cómo se muestra tiene que ver con la manera de pensar, esa manera de sacar conclusiones y de decidir qué hacer con la vida; se construyen o no proyectos, o se espera que la vida construya u ofrezca esos proyectos; y en ese esperar que la vida sea la que construya un proyecto, nunca se es libre, sino que se es un esclavo; un esclavo ya sea de los jefes, de los padres, de los profesores o lo peor, de los propios compañeros; es decir si pienso igual que todas las demás personas, entonces termino siendo manejado por aquellos que piensan un poco o muy diferente a mí, porque son ellos los que deciden los proyectos.

Una nueva manera de pensar, de construir las ideas, es la única manera de llegar a ser creativo; si se quiere ser creativo, se trata de romper con el pensar de todo el mundo, y aclaro que no se trata de pensar lo que piensen otros, sino de romper el cómo piensa la mayoría de la gente. La mayoría de la gente piensa de una manera que lo lleva a sacar conclusiones directamente, es decir convergentemente; pensar como todo el mundo piensa, básicamente sacar conclusiones sin el análisis suficiente, lleva a que en gran medida otros decidan por mí.
El pensamiento divergente obliga a un mayor análisis, a una profundización, a una valoración crítica de los hechos que se nos presentan, por lo tanto llegamos a conclusiones de más valor, y por ende nuestras decisiones van a terminar por ser asumidas con responsabilidad. Tendemos a no tener proyecto personal, sino que nuestro proyecto, el tuyo, el suyo etc., todos son iguales, porque aplicamos un modelo de pensamiento que no permite analizar, evaluar y criticar la realidad.

En las academias se fomenta el modelo de pensamiento convergente de muchas formas; una de ellas está en preparar al estudiante para el empleo no para el trabajo; a punta de repetición le hacen generar al estudiante la concepción de que lo que importa es emplearse, no trabajar. El profesor dice: ¿Pero qué es lo que está pensando? Cuando usted trabaje en la empresa, el jefe no le va a aceptar todos esos errores de ortografía. Y a base de repetir diálogos como éste, se crea la concepción de que la responsabilidad está en responder a un jefe, no a una profesión específica; eso termina en que la responsabilidad se perciba como responsabilidad con el jefe, y eso hace que se vuelen todos los parámetros de la ética por responderle a un jefe. El pensamiento divergente nos obligaría a cuestionar lo que estamos haciendo y las fases de lo que estamos haciendo.

\section{LA CREATIVIDAD Y LA INFORMACIÓN}

Memoria, capacidad para guardar información y sacarla, capacidad para guardar información y traerla al consciente; inteligencia, capacidad para relacionar información; creatividad, capacidad para generar productos, es decir nueva información, y genialidad, capacidad para evaluar y criticar los productos propios, es decir la nueva información, posibilitando el comienzo del ciclo nuevamente; la genialidad está en guardar información, relacionar ésta con la nueva información, crear otros productos, evaluarlos y ponerlos en funcionamiento como información.

Las percepciones que de las situaciones y de los hechos tengamos, y los recuerdos que nos hayan dejado esas percepciones, es lo que se conoce como información; por ende estamos hablando de vivencias, y éstas nacen de las acciones, por eso se hace necesario vivir con intensidad, y la intensidad sólo se consigue con una vida comprometida que permita sumar información. La vida está hecha de acciones, sino no sería perceptible, pero si la dejamos pasar sin enfrentarla y compartirla, nuestra memoria será limitada, perjudicando nuestras posibilidades de crear. 


\section{NIVELES DE LA CREATIVIDAD}

La divergencia es la que lleva a tener una búsqueda permanente, una actitud de creación, por eso los pensadores divergentes se irán especializando, y se irán haciendo más creativos en la medida que ejerciten las tres funciones básicas del pensamiento: MEMORIA, INTELIGENCIA y CREATIVIDAD, del uso que hagamos de ellas, nacen los niveles de la creatividad, que son: el primero es el nivel de la creatividad expresiva, básicamente una capacidad desarrollada de imitación, que nos hace capaces de imitar productos creativos, productos artísticos, productos de comunicación generados por otros, en ella se ha desarrollado una muy buena capacidad de observación y también una buena memoria; por dar un ejemplo de pensador divergente que se encuentre dentro este nivel de la capacidad creativa expresiva, hablemos de los tantos excelentes imitadores que aparecen en nuestra televisión, que manejan una buena capacidad de observación y de memorización, para poder establecer los giros que se producen en la rítmica de una voz, incluso en la expresión facial, en la expresión corporal de los personajes que imitan; éste es el nivel más bajo de la creatividad, sin embargo, ya allí estamos hablando de pensadores divergentes.

El segundo nivel es el de la productividad, allí se supera la imitación y se genera e inventa, pero sin romper la fuente original; es decir más claramente hay una imitación y una recreación; podríamos decir que muchos de los cultivadores de la comedia de situaciones, relatada en un escenario son un ejemplo válido de este nivel, pues ya allí han utilizado la generación, la imitación y la recreación que plantea nuevos elementos.

En el tercer nivel está la inventiva, en la que hay creación total, es decir nos encontramos con que lo que se inventa, como su nombre lo dice, es algo que nadie había creado; en muchas ocasiones nuestra creatividad no llega más allá del nivel expresivo, ya que lo único que podemos hacer es imitar; en el nivel de la inventiva generamos, es decir construimos algo que nadie había intentado antes; por ejemplo la historia de una pareja que al conocerse, descubren que fueron amantes en otras vidas.

El último de los niveles es el innovador, en el cual ya no sólo existe el nivel de creación, sino el nivel autocrítico, tanto de lo creado como de sus efectos sobre los medios a los cuales llega; es decir somos capaces de abstraernos de que el producto es hecho por nosotros mismos, y lo podemos criticar con suficiente fuerza, con suficiente profundi- dad, con suficiente basamento, y además podemos criticar el resultado que este producto tiene en el medio, en las personas a las cuales llegó; lógicamente este nivel es el que muy pocas personas serán capaces de alcanzar, porque es romper con la egolatría, es romper con aquello que todos creemos: "el que lo que hagamos está bien hecho, porque lo hemos hecho nosotros; y si el producto llega mal a un contexto, a un público, el que es bruto es el público, no nosotros", ese es el nivel innovador.

\section{LA ESPECIALIZACIÓN Y LA CREATIVIDAD}

Especialización no quiere decir conocer de una sola área del conocimiento, sino poder traer elementos de muchas áreas del conocimiento hacia la creación o desarrollo de un solo objeto. Este campo requiere de poder traer muchas áreas del conocimiento al análisis del producto y del resultado del producto en el entorno, en el contexto; es decir hemos hablado de algunos elementos que al concretarse en la fase innovadora y además especializada, nos están remitiendo a los genios; uno de los elementos que maneja el genio es la especialización, no la sectorización del conocimiento, es decir el genio es especializado en una sola cosa, aunque sabe sobre demasiados campos del conocimiento, todos los utiliza en beneficio del único objetivo específico de su especialización.

\section{Especialización no quiere decir conocer de una sola área del conocimiento, sino poder traer elementos de muchas áreas del conocimiento hacia la creación o desarrollo de un solo objeto.}

Si en la medicina, que es donde más entendemos las categorías de la especialización, somos especialista en el dedo gordo del pie derecho, no es que no sepamos sino del dedo gordo del pie derecho, no; es que sabemos de todo y cómo ese todo afecta o interviene en el funcionamiento del dedo gordo del pie derecho; sabemos del problema sanguíneo, es decir de los problemas de irrigación, por lo tanto sabemos del corazón, aunque ese conocimiento sólo lo aplique a solucionar el problema concreto, el funcionamiento del dedo gordo del pie derecho; sabemos de neurología, sabemos de dermatología porque ahí salen callos, sabemos del funcionamiento físico de los músculos, sabemos de huesos, etc.; no es que sepamos sólo de ese dedo gordo, es que sabemos sobre toda la medicina y lo aplicamos al dedo gordo; esa es nuestra función, aunque no solamente dentro del campo médico, para ser ese médico, debemos saber de física, porque el dedo gordo soporta 
una serie de fuerzas, y dentro de la física tendremos que saber de dinámica, por el movimiento; sabremos cómo la música, según el temperamento de la persona, ayuda a relajar o no el dedo gordo; y al hablar de temperamento se habla de psicología, por eso es inevitable que tengamos que saber cómo lo afecta el color o el tejido, porque aunque parece que el dedo siempre está a oscuras, el color interno y externo del zapato hará que sienta más o menos calor, etc.

El genio es el que llega a descubrir este último nivel, básicamente porque conoce de tantas áreas que, en realidad, puede hacer un verdadero análisis de los productos y de los procesos, aún de los propios; como sabe de tantas cosas, él tiene una gran capacidad de evaluar y criticar.

\section{CONCLUSIÓN}

Ya sabemos que la creatividad sin problemas no existe; es decir si tendemos a evitar el enfrentar los problemas, de creativos no tendremos absolutamente nada, seremos los pensadores más convergente, por eso se puede decir que el pensamiento convergente frena, estanca la creatividad de cada persona totalmente; cuando vivimos por y para las normas la creatividad desaparece, se anula en gran medida la inteligencia, funciona en algo la memoria y nos acercamos a la brutalidad, al pensamiento automático, simplemente dando respuesta a los momentos que se viven. Por eso es tan importante que cada uno logre analizar hasta dónde es convergente, y se proponga despertar todas aquellas funciones del pensamiento que todos tenemos, pero que en el transcurso de nuestra vida van siendo anuladas, precisamente por toda la normatividad que nos inventamos o nos imponen, normatividad en la cual pueden estar los prejuicios. A quienes su ambiente les coarta las posibilidades de crear se anularon, y eso no está dado exclusivamente por la pobreza, pues los prejuicios pueden hacer mucho más daño que la pobreza.

La creatividad no tiene que ver con el alto o bajo coeficiente intelectual, pero si la inteligencia es el arte de razonar, cuando se es creativo se tiene que razonar, así que ahí está presente la inteligencia, y si razono más, puede que llegue a ser más creativo, por eso es que la creatividad está familiarizada con la inteligencia. La inteligencia apoyará más la creatividad, pero se puede ser creativo sin tener tanta inteligencia, éste es el nivel de la recursividad, con el cual solucionamos simplemente nuestras necesidades concretas reales o inventadas.

Tratemos de identificar nuestras necesidades espirituales, ejercitemos nuestra memoria, nuestra inteligencia y nuestra creatividad, así comenzaron todos los genios. 